\title{
EVALUACIÓN DE LA SOBREVIVENCIA DE Steneotarsonemus spinki EN PLANTAS HOSPEDANTES
}

\author{
Jean Alexander Gamboa Tabares ${ }^{1}$, Ruth León González², Víctor Manuel Cartín Leiva ${ }^{3}$ \\ José Francisco Álvarez Bonilla ${ }^{4}$, Israel Garita Cruz ${ }^{5}$
}

\begin{abstract}
RESUMEN
El ácaro del arroz Steneotarsonemus spinki Smiley se encontró en Costa Rica en el año 2004, como parte del complejo de organismos causantes del vaneado de la panícula y la pudrición de la vaina de arroz. Con el fin de evaluar la sobrevivencia de este ácaro en las plantas hospedantes Oryza sativa, Oryza latifolia y Echinochloa colona, se desarrollaron dos estudios bajo condiciones de invernadero e in vitro durante los meses de octubre y noviembre de 2007, en la Hacienda La Ligia, ubicada en la región arrocera Pacífico Central de Costa Rica. Se estudió en invernadero la sobrevivencia, crecimiento poblacional y estructura poblacional a partir de infestaciones, directa y voluntaria del ácaro. En el ensayo de invernadero se realizaron muestreos durante 18 días cada 24 horas con ayuda de un estereoscopio, y se cuantificó el número de individuos de $S$. spinki encontrados por cada etapa de desarrollo en cada especie. El ácaro completó su ciclo de vida en O. sativa y O. latifolia, y en la especie arvense E. colona solo se observaron hembras, huevos y larvas móviles. Se obtuvieron tres tasas de sobrevivencia: TE (Tasa de establecimiento): 1,70 y 0,79; TFS (Tasa finita de sobrevivencia): 3,54 y 4,53; TIS (Tasa instantánea de sobrevivencia): 1,08 y 1,34; para 0. sativa y O. latifolia, respectivamente; y dos tasas de crecimiento poblacional: $r 1$ (Tasa media de crecimiento): 18,19 y 22,51; r2 (Tasa de crecimiento per capita): 0,60 y 0,69; para O. sativa y O. latifolia, respectivamente. El ácaro tuvo un crecimiento poblacional de tipo exponencial durante el período de evaluación. En el ensayo in vitro se realizaron muestreos cada 12 horas y se obtuvo una duración media del ciclo de vida de $S$. spinki desde huevo hasta adulto de siete días a temperatura de $25^{\circ} \mathrm{C}$. La mortalidad durante el ciclo de vida fue de $62 \%, 74 \%$ y 100 $\%$, para O. sativa, O. latifolia y E. colona, respectivamente. Se concluyó que S. spinki solo se presenta como planta hospedante alterna en los campos de arroz en Costa Rica, la especie arvense $O$. latifolia; y $E$. colona es una planta hospedante ocasional que permite el refugio transitorio por cortos períodos de tiempo.
\end{abstract}

Palabras clave: Oryza sativa, Oryza latifolia, Echinochloa colona, crecimiento, estructura poblacional, invernadero.

\footnotetext{
1 Tesis sometida a consideración del Tribunal Examinador del Sistema de Estudios de Posgrado de la Universidad Nacional para optar al grado de Magíster Scientiae en Agricultura Alternativa con mención en Agricultura Ecológica. Docente-investigador Universidad de la Amazonia. Correo electrónico: gamboatabares@gmail.com

2 Instituto Nacional de Investigación e Innovación y Transferencia en Tecnología Agropecuaria (INTA), Correo electrónico: rleon@ inta.com Dpto. Investigación e Innovación Teléfono: (506)2231- 5055.

3 Escuela de Ciencias Agrarias, Universidad Nacional. Teléfono: (506)2277-3298. Correo Electrónico: vcartin@una.ac.cr

4 Equipo de Extensión Agrícola. Dirección Regional Chorotega. Ministerio de Agricultura y Ganadería. Liberia, Guanacaste, Costa Rica. Teléfono: (506)2666-0261

5 Gerente de Investigación Duwest Inc. Centroamérica y Caribe Correo electrónico: Israel.Garita @ duwest.com. Apartado postal: 219-3009 Santa Bárbara de Heredia, Costa Rica.
} 


\section{INTRODUCCIÓN}

El ácaro Steneotarsonemus spinki es una especie oriunda del sudeste asiático (Ramos y Rodríguez 1997) que se señaló por primera vez en Costa Rica en el año 2004 (Barquero 2004), como una plaga del cultivo del arroz (Oryza sativa L.). Se encuentra asociado estrechamente al patógeno fúngico Sarocladium oryzae Sawada (Sandoval et al. 1998). Ambos organismos forman el complejo causante del vaneado de la panícula y la pudrición de la vaina de arroz, el cual ha provocado pérdidas en Costa Rica de hasta el $100 \%$ en algunas áreas (Correa-Victoria 2006).

Las medidas de combate del ácaro implementadas en diferentes países, han sido dirigidas al manejo adecuado del cultivo y uso de variedades resistentes. El control biológico aunque potencialmente útil, no ha podido ser adoptado debido a diferentes problemas en el manejo y eficiencia de los productos utilizados, así como aspectos relacionados con el ciclo de vida del ácaro, su ubicación en la planta, y las altas poblaciones desarrolladas. Se ha informado que el combate químico sobre las poblaciones del ácaro es bajo, y la mayoría de los productos son altamente tóxicos a los enemigos naturales. En general, los ácaros desarrollan rápidamente resistencia a los acaricidas, por lo que se recomienda utilizar diferentes productos y de forma alterna. El manejo cultural sigue siendo el método más eficiente para el control del ácaro (CorreaVictoria 2006).

Dentro de las prácticas culturales eficientes en los programas de manejo del ácaro, se encuentran la eliminación de los residuos de cosecha y de plantas arvenses asociadas a los campos de arroz. En campos arroceros de Costa Rica, se ha determinado la presencia de S. spinki en las especies arvenses Oryza latifolia, Echinochloa colona, Eleusine indica y Rottboellia cochinchinensis. Hasta la fecha, no han sido realizados estudios similares que permitan determinar la relación de estas especies hospedantes, con respecto a la sobrevivencia del ácaro.
Con respecto a la biología y comportamiento poblacional de $S$. spinki, se sabe que el ciclo de vida incluye, al igual que en el resto de los tarsonémidos, la presencia de tres estadios: huevo, larva (comprendiendo un período de quiescencia) y adulto, así como la existencia de un mecanismo sexual haplo-diploide. Chen et al. (1979) plantean además que la duración del ciclo de vida en condiciones de laboratorio disminuye con el incremento de la temperatura, y que de igual forma ocurre para el período de puesta y la longevidad, la que es mayor en la hembra adulta. Santos et al. (1998) indicaron una duración del ciclo de vida (huevo a adulto) de 5,1 días en condiciones ambientales de $290 \mathrm{C}$, la que debe ser similar bajo las condiciones de la región arrocera Pacífico Central en Costa Rica.

Consideraciones de Almaguel et al. (2003), en cuanto a la estructura de población, incluyen que $S$. spinki en $O$. sativa presenta una tendencia similar para todas las edades. El aumento al final del ciclo del cultivo se explica por la relación nominal entre machos y hembras y con la población total; donde se observa que el incremento en la población adulta ocurre antes en las hembras (dos hembras por macho) y después en los machos $(0,2$ hembras por cada macho) y disminuyen los inmaduros. Esto reafirma el poder migratorio de las hembras y su fertilidad (arrenotoquia); máxima antes de ese intervalo, para además garantizar la reproducción sexual de los emigrantes como estrategia de sobrevivencia y perpetuación de la especie.

Teniendo en cuenta la necesidad de profundizar en aspectos relacionados con la biología del ácaro sobre posibles especies hospedantes alternas, se ha planteado como objetivo de investigación, evaluar la sobrevivencia, crecimiento y estructura poblacional de S. spinki en las plantas arvenses O. latifolia y E. colona; y la variedad de arroz comercial $O$. sativa var. Palmar 18. Lo anterior, permitirá determinar la relación de cada especie hospedante con los problemas provocados por el ácaro, bajo las condiciones agroecológicas de las zonas arroceras de Costa Rica. 


\section{MATERIALES Y MÉTODOS}

La evaluación de la sobrevivencia de $S$. spinki en tres plantas hospedantes ( $O$. sativa, $O$. latifolia y E. colona) se realizó durante los meses de octubre (invernadero) y noviembre (in vitro) de 2007. Los experimentos se establecieron en la Hacienda La Ligia, ubicada en la provincia de Puntarenas, cantón Parrita, distrito La Julieta. La región arrocera Pacífico Central, presenta precipitación promedio de $3000 \mathrm{~mm}$ anuales, altitud entre $0-100$ m.s.n.m, temperatura de $25^{\circ} \mathrm{C}$ y humedad relativa de $85 \%$; ubicándose en la zona de vida bosque húmedo tropical transición a perhúmedo (Holdridge 1993).

\section{Sobrevivencia bajo condiciones de invernadero}

El experimento se realizó en el invernadero de la Hacienda La Ligia bajo condiciones ambientales de humedad relativa (85\%) y temperatura $\left(28^{\circ} \mathrm{C}\right)$ de la región. Se utilizaron 720 bolsas, con dimensiones de $15 \mathrm{~cm}$ de altura y $12 \mathrm{~cm}$ de diámetro, capacidad para $1,5 \mathrm{~kg}$ de sustrato, conteniendo las tres fuentes alimenticias (tratamientos) a evaluar: Oryza sativa L. var. Palmar 18, Oryza latifolia y Echinochloa colona.

Cada tratamiento consistió de 240 plantas en estado de primordio de la panícula (conocido como estado de embuchamiento), recolectadas en campos de arroz de la Hacienda La Ligia, verificados previamente sin presencia de S. spinki. El sustrato que se utilizó fue suelo extraído con cada planta de campos de arroz. El experimento tuvo un diseño de bloques completos al azar, con tres tratamientos y cuatro repeticiones. Cada parcela experimental estuvo constituida por 60 plantas (seis plantas $\times 10$ plantas) separadas entre parcelas a $1,4 \mathrm{~m}$ y entre bloques a 0,8 $\mathrm{m}$, equivalente a una superficie de $33,3 \mathrm{~m}^{2}$. La unidad de muestreo fue de dos plantas contenidas en igual número de bolsas.

En el estudio se realizó infestación directa y voluntaria del ácaro, por lo que cada una de las plantas fue objeto de dos procedimientos: 1) se tomaron plantas de arroz, se extrajeron vainas de hojas altamente infestadas por $S$. spinki y se colocaron a cada planta (en la lígula de la hoja bandera) del experimento, a tres centímetros de éstas (15 - 20 ácaros/ trozo vaina); 2) se colocaron al azar 10 plantas/ parcela experimental, altamente infestadas por S. spinki, para facilitar infestación voluntaria.

El riego fue realizado diariamente entre 6:00 y 8:00 am y entre 5:00 pm y 6:00 pm, con regadera manual. Se tuvo en cuenta durante cada riego, dejar las macetas inundadas para favorecer crecimiento de las plantas.

Se realizaron observaciones durante 18 días cada 24 horas (entre 10:00 am y 1:00 pm) con estereoscopio (Olympus Tokio Japan 219719) a 20X, y se hizo el conteo de individuos de S. spinki por cada etapa de desarrollo en dos plantas (tres secciones de vainas de hojas de tres centímetros a cada planta) por parcela experimental. Se cuantificó el número de individuos por estadio de desarrollo (huevos, estados inmaduros, adultos hembras y machos) en cada una de las plantas indicadas. La determinación de plantas hospedantes alternas de $S$. spinki se realizó de acuerdo con las condiciones óptimas de hábitat y alimentación ofrecidas por cada especie arvense, que permitió el desarrollo normal del ciclo de vida y la sobrevivencia del ácaro.

\section{Sobrevivencia}

Para el análisis de la sobrevivencia de S. spinki en cada planta hospedante que se evaluó, se tuvieron en cuenta los siguientes estimadores: 
Tasa de establecimiento (Kang-Chen y ChyiChen 1979)

$\mathrm{TE}=\mathrm{Nhu} / \mathrm{Nhe}$

Donde:

TE: Tasa de establecimiento

Nhu: Número huevos en un período de tiempo dado

Nhe: Número hembras en un período de tiempo dado

Tasa finita de sobrevivencia (Anónimo 2005).

$\mathrm{TFS}=\mathrm{Pn} / \mathrm{P} 1$

Donde:

TFS: Tasa finita de sobrevivencia

Pn: Tamaño final de la población

P1: Tamaño inicial de la población

Tasa instantánea de sobrevivencia (Anónimo 2005)

$\mathrm{TIS}=\ln (\mathrm{Pn} / \mathrm{P} 1)$

Donde:

TIS: Tasa instantánea de sobrevivencia

Pn: Tamaño final de la población

P1: Tamaño inicial de la población

\section{Crecimiento Poblacional}

El análisis de crecimiento poblacional de $S$. spinki en cada planta hospedante evaluada, incluyó los siguientes estimadores:

\section{Tasa media de crecimiento (\%) (FAO 2006)}

La tasa de crecimiento, $r$ (expresada en porcentaje), se calcula entre dos puntos de la serie cronológica usando la siguiente fórmula:

$r 1=[\ln (P n / P 1) / n] \times 100$

Donde:

r1: Tasa de crecimiento
Pn: Tamaño final de la población

P1: Tamaño inicial de la población

n: Número de días que compone el período

Tasa de crecimiento per cápita (FAO 2006)

$r 2=(P n-P 1) / P n$

Donde:

r2: Tasa de crecimiento per cápita

Pn: Tamaño final de la población

P1: Tamaño inicial de la población

\section{Sobrevivencia In Vitro}

El experimento se realizó in vitro bajo condiciones ambientales de humedad relativa ( $85 \%$ ) y temperatura (25\% C) en la Hacienda La Ligia. Las unidades de cría fueron colocadas en una caja de cartón con $100 \%$ de oscuridad, cuyo propósito fue simular las condiciones de oscuridad presentadas en las vainas de hojas de plantas de la familia Poaceae.

\section{Recolecta y determinación de la especie}

Adultos del ácaro fitófago $S$. spinki fueron colectados en vainas de hojas de Oryza sativa variedad Palmar 18, en campos comerciales de la Hacienda La Ligia, ubicada en la región arrocera Pacífico Central. Las hojas se observaron bajo el microscopio estereoscópico para seleccionar los ácaros fitófagos, de los que fueron extraídos distintos ejemplares adultos para machos y hembras, y se colocó cada ejemplar en láminas microscópicas permanentes. La clave taxonómica de Smiley et al. (1993) fue utilizada para la identificación de la especie.

\section{Ciclo de vida de S. spinki}

La obtención de huevos del ácaro se realizó en diez cápsulas de Petri $(8,9 \mathrm{~cm}$ de diámetro por $1,8 \mathrm{~cm}$ de alto), previamente se prepararon con algodón humedecido. Se colocaron 50 individuos (hembras y machos) en cada unidad de cría. Transcurridas 12 horas, las unidades de cría fueron observadas al estereoscopio para determinar el número de huevos/ unidad de cría, hasta obtener un total de 360 huevos. 
El estudio del ciclo de vida de S. spinki se llevó a cabo en unidades de cría similares, con algodón de $0,5 \mathrm{~cm}$ de espesor, previamente humedecido para mantener la turgencia de las hojas. Sobre cada unidad de cría se colocó una sección de nudo y vaina de hojas de cada planta evaluada, con la cara interna hacia arriba y rodeado cada nudo por una banda de algodón humedecido. Para evitar el escape de los ácaros, se colocó en los bordes de cada vaina pequeñas porciones de vaselina.

Se utilizó un diseño completamente aleatorizado, que consistió en tres tratamientos y cinco unidades de cría (repeticiones) para cada una de las fuentes alimenticias evaluadas. Se colocó en cada unidad experimental, diez huevos de $S$. spinki, y posteriormente fueron observados periódicamente cada $12 \mathrm{~h}$ para determinar el tiempo de duración de cada una de las etapas del ciclo de vida (huevo hasta adulto) del ácaro.

Las variables evaluadas en cada unidad experimental fueron: número de huevos, número de larvas móviles, número de larvas inactivas, machos adultos y hembras adultas. Igualmente se registró para cada etapa de desarrollo, el número de individuos muertos.

\section{RESULTADOS}

\section{Sobrevivencia bajo condiciones de invernadero}

\section{Sobrevivencia}

Se evaluó durante 18 días la sobrevivencia de $S$. spinki y su crecimiento poblacional sobre $O$. sativa, $O$. latifolia y $E$. colona. Con respecto a la colonización por parte del ácaro, los resultados obtenidos para la especie $O$. sativa, indicaron que durante los primeros tres días se obtuvieron entre tres y seis ácaros por planta, los cuales correspondían a individuos adultos hembras. Se observó en el mismo período, menor número de hembras en $O$. latifolia, entre uno y cuatro individuos/ planta; y muy mínima la colonización en E. colona con promedio de una hembra por planta.
El aumento poblacional de S. spinki en las tres plantas evaluadas se muestra en las Figuras 1,2 y 3 . En 0 . sativa se contabilizaron durante todo el período de evaluación un total de 4787 individuos, de los que corresponde el $44,1 \%$ a huevos, 27,6 \% a estados inmaduros, el 23,6 a hembras adultas y el 4,6 \% a machos. La densidad máxima que se alcanzó para S. spinki fue de 163 ácaros/ planta en el día 18 de evaluación y la mínima de 1,1 ácaros/ planta en los primeros tres días donde se dio la colonización por parte del ácaro.

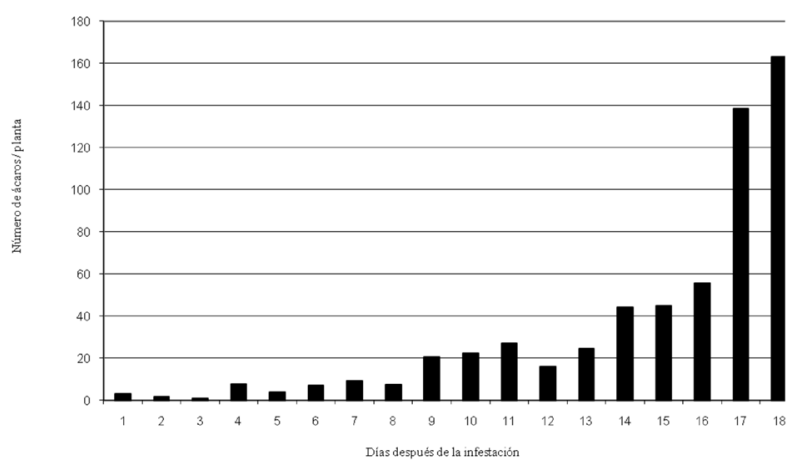

Figura 1. Densidad poblacional de S. spinki (número de individuos de todos los estadios/ planta) en plantas de $O$. sativa evaluadas bajo condiciones de invernadero en la Hacienda La Ligia, Puntarenas, Costa Rica. 2007.

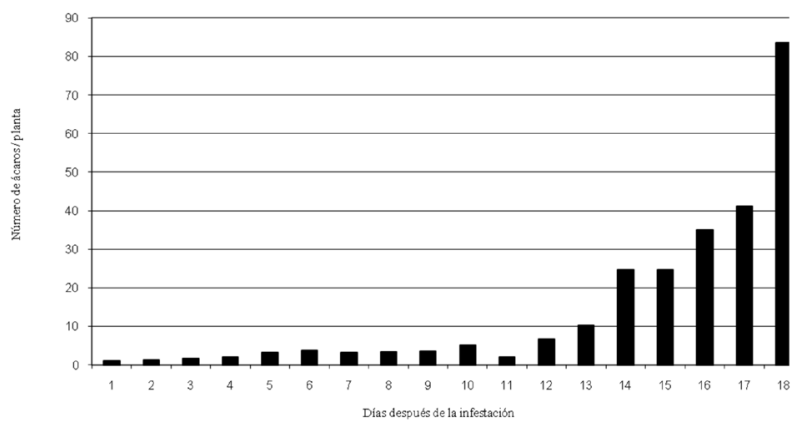

Figura 2. Densidad poblacional de S. spinki (número de individuos de todos los estadios/ planta) en plantas de $O$. latifolia evaluadas bajo condiciones de invernadero en la Hacienda La Ligia, Puntarenas, Costa Rica. 2007. 


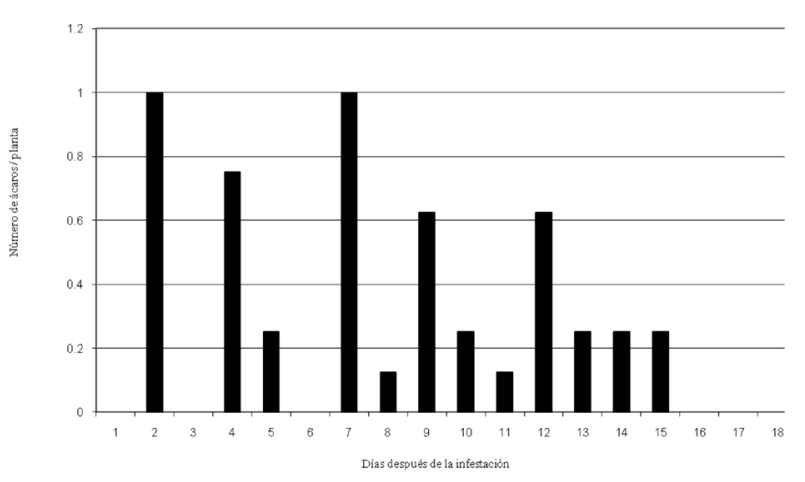

Figura 3. Densidad poblacional de S. spinki (número de individuos de todos los estadios/ planta) en plantas de E. colona evaluadas bajo condiciones de invernadero en la Hacienda La Ligia, Puntarenas, Costa Rica. 2007.

Se contó un total de 2050 individuos en la especie O. latifolia, de los que el 42,2 \% correspondió a huevos, 29,9 \% a hembras, 32,7 \% a estados inmaduros y $4,2 \%$ a machos. La densidad máxima alcanzada fue de 83,5 ácaros/ planta y la mínima de 1,13 ácaros/ planta.

En la especie E. colona se contabilizaron 44 individuos S. spinki, los que correspondieron a 29 hembras, 12 huevos y tres estados inmaduros en el estadio correspondiente a larva móvil. El mayor número de individuos observado fue de dos hembras y tres huevos en una planta.

Los resultados obtenidos con respecto a la calidad de las plantas evaluadas como hospedantes de $S$. spinki, indicaron que el ácaro solamente completa su ciclo de vida en las especies $O$. sativa y $O$. latifolia. Se presentaron diferencias en la densidad poblacional, donde $O$. latifolia solo hospedó el $42 \%$ de la población total que se encontró en $O$. sativa. En la especie E. colona las hembras colonizaron en muy bajo número, colocaron pocos huevos, y las larvas móviles que emergieron no consiguieron llegar al estadio de larva inactiva.

Se obtuvieron tres tasas de sobrevivencia para $S$. spinki en las dos fuentes alimenticias $O$. sativa y O. latifolia (Cuadro 1). Es de mencionar que teniendo en cuenta el comportamiento poblacional de $S$. spinki, durante todo el ensayo, las tasas fueron calculadas para tres períodos definidos de tiempo: 1-6, 7-12 y 1318 días después de infestación.

La tasa de establecimiento fue una función de la reproducción y de la dispersión, y relacionó el número de huevos con el número de hembras presentes en una planta. Se observó que $S$. spinki presentó una menor tasa de establecimiento en $O$. latifolia que en $O$. sativa, lo que igualmente correspondió con la diferencia de la población acumulada encontrada en la planta arvense.

La tasa finita de sobrevivencia relacionó el tamaño final de la población con respecto al tamaño inicial, en períodos de tiempo dados. Esta relación indicó que aunque en $O$. sativa se observó en general una mayor población, el aumento proporcional de la población final con respecto a la inicial, fue mayor en 0 . latifolia. Similares resultados fueron obtenidos para la tasa instantánea de sobrevivencia.

Cuadro 1. Tasas de sobrevivencia de S. spinki en dos fuentes alimenticias bajo condiciones de invernadero en la Hacienda La Ligia, Puntarenas, Costa Rica. 2007.

\begin{tabular}{|c|c|c|c|c|c|c|}
\hline \multirow[b]{2}{*}{$\begin{array}{c}\text { Períodos de } \\
\text { evaluación (días) }\end{array}$} & \multicolumn{2}{|c|}{$\begin{array}{c}\text { Tasa de } \\
\text { establecimiento }\end{array}$} & \multicolumn{2}{|c|}{$\begin{array}{l}\text { Tasa finita de } \\
\text { de sobrevivencia }\end{array}$} & \multicolumn{2}{|c|}{$\begin{array}{l}\text { Tasa instantánea } \\
\text { de sobrevivencia }\end{array}$} \\
\hline & $\begin{array}{c}0 . \\
\text { sativa }\end{array}$ & $\begin{array}{c}0 . \\
\text { latifolia }\end{array}$ & $\begin{array}{c}0 . \\
\text { sativa }\end{array}$ & $\begin{array}{c}0 . \\
\text { latifolia }\end{array}$ & $\begin{array}{c}0 . \\
\text { sativa }\end{array}$ & $\begin{array}{c}0 . \\
\text { latifolia }\end{array}$ \\
\hline $1-6$ & 1,0 & 0,2 & 2,3 & 3,3 & 0,8 & 1,2 \\
\hline $7-12$ & 2,2 & 0,3 & 1,8 & 2,1 & 0,6 & 0,8 \\
\hline $13-18$ & 1,9 & 1,9 & 6,6 & 8,1 & 1,9 & 2,1 \\
\hline Media & 1,7 & 0,8 & 3,5 & 4,5 & 1,1 & 1,3 \\
\hline $\mathrm{DS}^{*}$ & 0,6 & 1,0 & 2,7 & 3,2 & 0,7 & 0,7 \\
\hline
\end{tabular}

*DS: Desviación estándar

Se obtuvo una población total acumulada de 598,4 individuos/ planta para O. sativa (Figura 4). El mayor número de individuos / planta se obtuvo al final del ensayo con promedio de 163,0 , del que $46,9 \%$ corresponden a huevos, $30,8 \%$ estados inmaduros, $14,9 \%$ adultos hembras y $7,4 \%$ adultos machos. Las hembras y huevos aparecieron desde el primer día de muestreo, los estados inmaduros a partir del quinto día y adultos machos a partir del día 11 (Figura 4). 


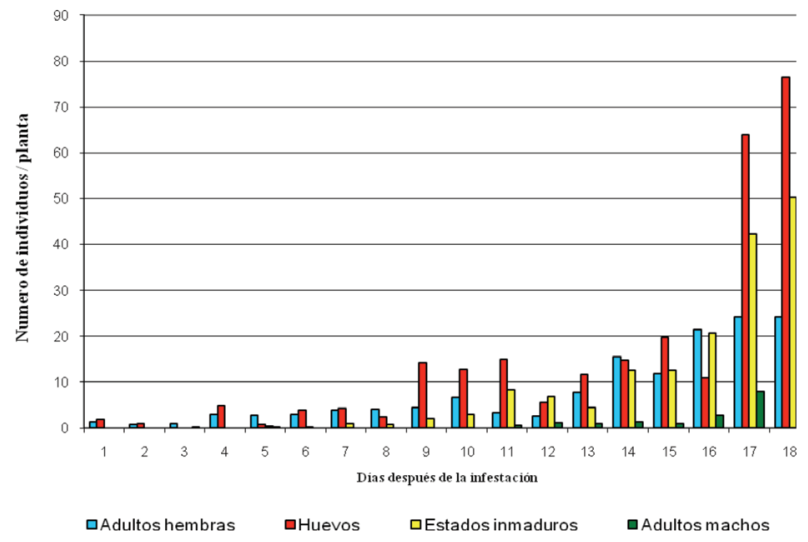

Figura 4. Densidad poblacional de adultos, estados inmaduros y huevos $S$. spinkien $O$. sativa bajo condiciones de invernadero en la Hacienda La Ligia, Puntarenas, Costa Rica. 2007.

Se cuantificó en la especie arvense $O$. latifolia, una población acumulada de 256,3 individuos/ planta y un promedio a los 18 días de muestreo de 83,5 individuos/ planta (Figura 5). Al final del período la población que se encontraba estaba representada en un $49,6 \%$ de huevos, $26,6 \%$ estados inmaduros, 21,4\% hembras y $2,4 \%$ machos. Las hembras colonizaron la especie en los primeros tres días. A partir del cuarto día empezaron a aparecer huevos; los estados inmaduros a partir del sexto día y alta presencia de machos solo fue observada a partir del día 12.

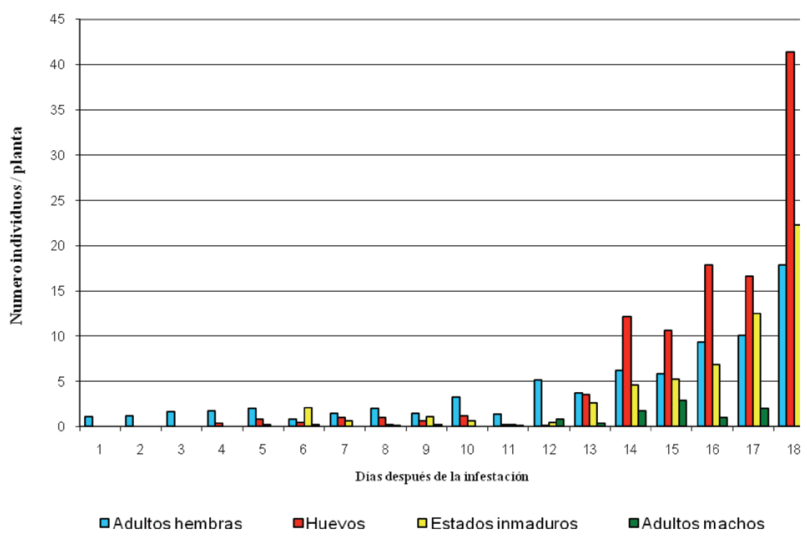

Figura 5. Densidad poblacional de adultos, estados inmaduros y huevos S. spinki en $O$. latifolia bajo condiciones de invernadero en la Hacienda La Ligia, Puntarenas, Costa Rica. 2007.
En la planta arvense E. colona la colonización por parte de las hembras de S. spinki presentó una densidad de un individuo/ planta. Se observaron huevos a partir del cuarto día de colonización con promedio máximo de 0,4 huevos/ planta, y en el décimo segundo día aparecieron escasos individuos en estado de larva móvil, los que finalmente murieron posiblemente por las insuficientes condiciones nutricionales ofrecidas por la especie arvense al ácaro. Las larvas móviles que se observaron se movían rápidamente, empezaron a tener apariencia de desecación y finalmente murieron. La población total acumulada de ácaros observados en la especie fue de 44 individuos en 144 plantas evaluadas durante el período de muestreo (Figura 6).

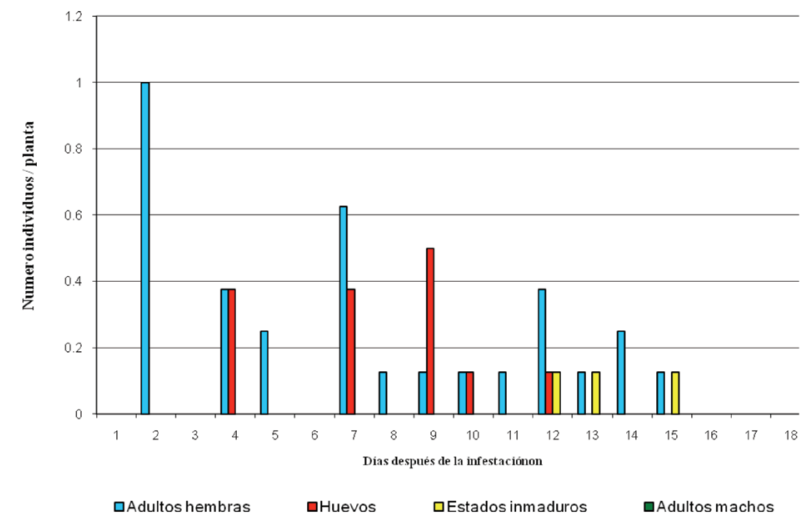

Figura 6. Densidad poblacional de adultos, estados inmaduros y huevos S. spinki en E. colona bajo condiciones de invernadero en la Hacienda La Ligia, Puntarenas, Costa Rica. 2007.

Las plantas que se utilizaron de $O$. sativa, $O$. latifolia y E. colona para la evaluación de la sobrevivencia de $S$. spinki, se encontraban al momento de infestación en la etapa de primordio de la panícula. Se pudo observar que para el día 12, después de infestación, el $75 \%$ de plantas de la especie O. latifolia habían alcanzado la etapa de llenado de grano. El $25 \%$ de las plantas de las especies O. sativa y E. colona alcanzaron esta etapa el día 18 después de infestadas. El número de ácaros por planta cuantificados, indicaron que para el día 18, la especie O. latifolia alcanzó 
el $51,2 \%$ de la población encontrada para $\mathrm{O}$. sativa.

Solo 0 . sativa y $O$. latifolia ofrecieron las condiciones de hábitat y alimenticias para la culminación del ciclo de vida del ácaro S. spinki. Es por lo anterior que los análisis de crecimiento y estructura poblacional se realizan solamente para estas dos especies.

\section{Crecimiento poblacional}

Para el análisis del crecimiento poblacional, se tomaron como referencia tres períodos dentro de la evaluación total realizada. El primer período fue determinado entre los días uno y seis, el segundo entre los días 7 y 12 y el tercero entre los días 13 y 18 de muestreo. Las tasas de crecimiento que se obtuvieron en los tres períodos de análisis, para las dos fuentes alimenticias $O$. sativa y $O$. latifolia, fueron levemente mayores para la especie arvense (Cuadro 2).

Cuadro 2. Tasas de crecimiento de S. spinki en dos fuentes alimenticias evaluadas bajo condiciones de invernadero en la Hacienda La Ligia, Puntarenas, Costa Rica. 2007.

\begin{tabular}{|c|c|c|c|c|}
\hline \multirow[b]{2}{*}{$\begin{array}{l}\text { Períodos de } \\
\text { evaluación (días) }\end{array}$} & \multicolumn{2}{|c|}{$\begin{array}{c}\text { Tasa de } \\
\text { crecimiento (\%) }\end{array}$} & \multicolumn{2}{|c|}{$\begin{array}{c}\text { Tasa de } \\
\text { crecimiento per cápita }\end{array}$} \\
\hline & $\begin{array}{c}0 . \\
\text { sativa }\end{array}$ & $\begin{array}{c}0 . \\
\text { latifolia }\end{array}$ & $\begin{array}{c}0 . \\
\text { sativa }\end{array}$ & $\begin{array}{c}0 . \\
\text { latifolia }\end{array}$ \\
\hline $1-6$ & 13,7 & 20,1 & 0,6 & 0,7 \\
\hline $7-12$ & 9,4 & 12,5 & 0,4 & 0,5 \\
\hline $13-18$ & 31,5 & 35,0 & 0,8 & 0,9 \\
\hline Media & 18,2 & 22,5 & 0,6 & 0,7 \\
\hline DS & 11,7 & 11,4 & 0,2 & 0,2 \\
\hline
\end{tabular}

La tasa media de crecimiento expresada en porcentaje, indicó que el período de mayor incremento poblacional en las dos especies evaluadas, se dio entre los días 13 y 18 después de la colonización. La población de S. spinki aumentó en $O$. sativa un $31,5 \%$ con respecto a la población inicial que se tenía en cada planta; y en 0 . latifolia aumentó $35,0 \%$. En el mismo período, la tasa de crecimiento per cápita indicó que $O$. sativa por cada individuo presente en la población inicial, hubo un incremento de 0,8 individuos, y en $O$. latifolia de 0,9 individuos.

Las figuras de dispersión poblacional (Figura 7) indicaron que $S$. spinki tuvo un crecimiento poblacional de tipo exponencial. Se observó en todos los momentos de muestreo, mayor número de individuos en $O$. sativa, y tasas de crecimiento levemente mayores para $O$. latifolia durante los períodos analizados.
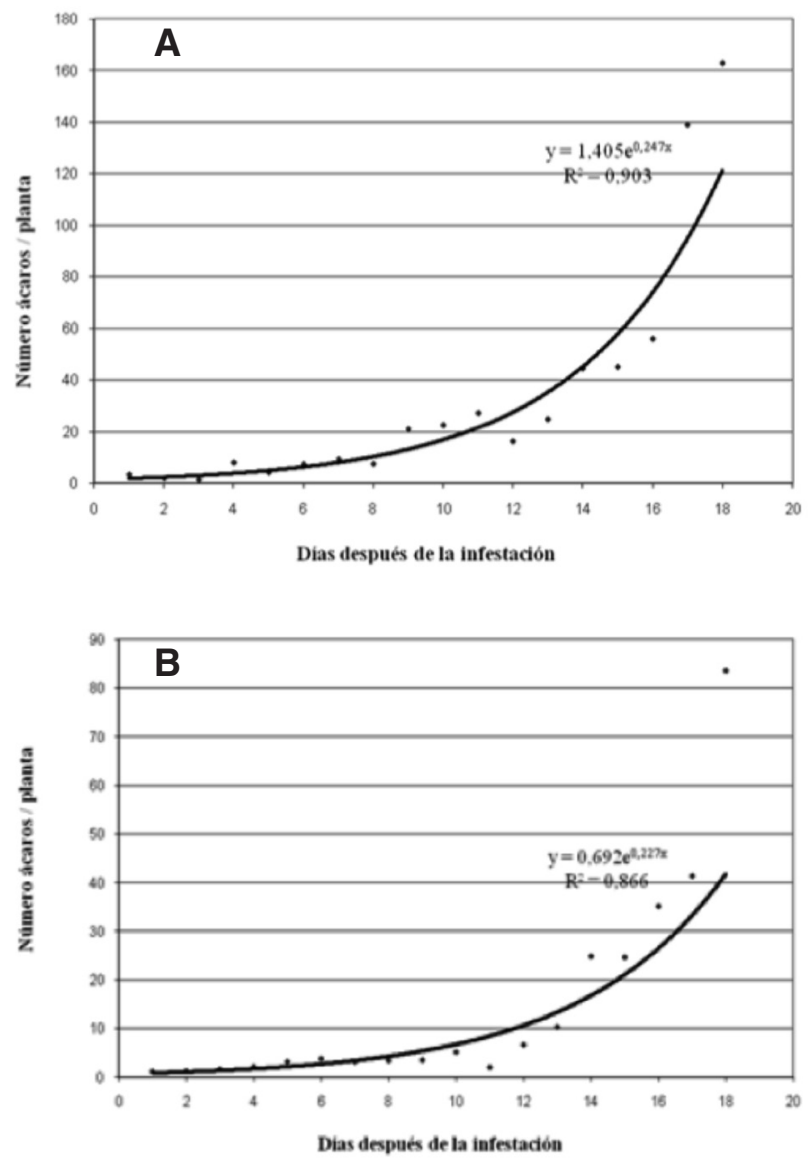

Figura 7. Crecimiento poblacional de $S$. spinki, en plantas arvenses (A) $O$. sativa), (B) $O$. latifoli bajo condiciones de invernadero en la Hacienda La Ligia, Puntarenas, Costa Rica. 2007.

\section{ESTRUCTURA POBLACIONAL}

La estructura poblacional indicó que durante los tres primeros días de colonización de $S$. spinki en $O$. sativa, la presencia de hembras adultas representó hasta un $88,9 \%$ del 
número total de individuos/ planta (Figura 8) y el porcentaje restante correspondió a huevos. No hubo presencia de machos adultos, lo que indicó que la colonización de nuevas plantas fue realizada solamente por las hembras. La mayor proporción de huevos se obtuvo en el día noveno, con un 68,7 \% con respecto a la población total encontrada. En el día 12 de muestreo, los estados inmaduros tuvieron el mayor porcentaje con respecto a la población total, con $42,2 \%$. Los machos adultos aparecen a partir del día 11, pero su mayor proporción poblacional ocurrió en el día 18, con el $7,4 \%$.

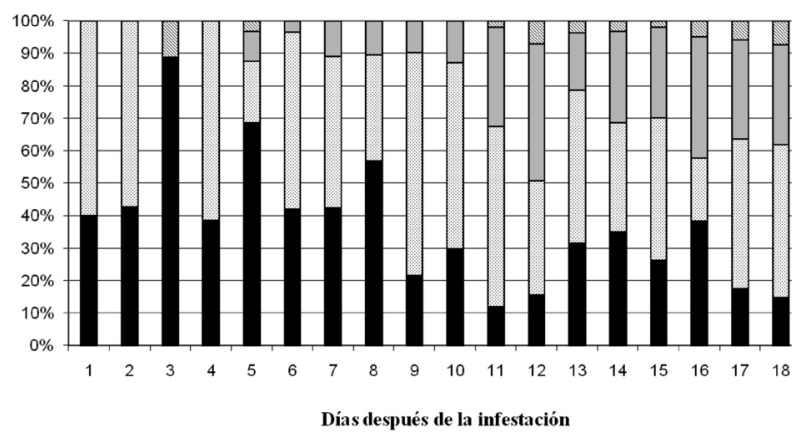

- Adultoshembras aHuevos aEstadosinmaduros aAdultos machos

Figura 8. Estructura poblacional de S. spinki en $O$. sativa bajo condiciones de invernadero en la Hacienda La Ligia, Puntarenas, Costa Rica. 2007.

En O. latifolia la estructura poblacional se caracterizó de la siguiente manera (Figura 9) durante los primeros tres días de muestreo, el $100 \%$ de la población fueron hembras, en el día 18 la presencia de éstas solo representó el $21,4 \%$ de la población total. En el día 7 se obtuvo que el $29,6 \%$ de la población total eran huevos, y durante los días 14 a 18 hubo un aumento considerable de esta proporción hasta alcanzar el 50,9\%. El mayor porcentaje correspondiente a estados inmaduros fue de 30,3, y se obtuvo en el día 17 de muestreo. La aparición de machos adultos se dio a partir del día 6, y la mayor densidad y participación poblacional se presentó en el día 15 con 2,9 individuos/ planta, y 11,7 \% de la población total encontrada.

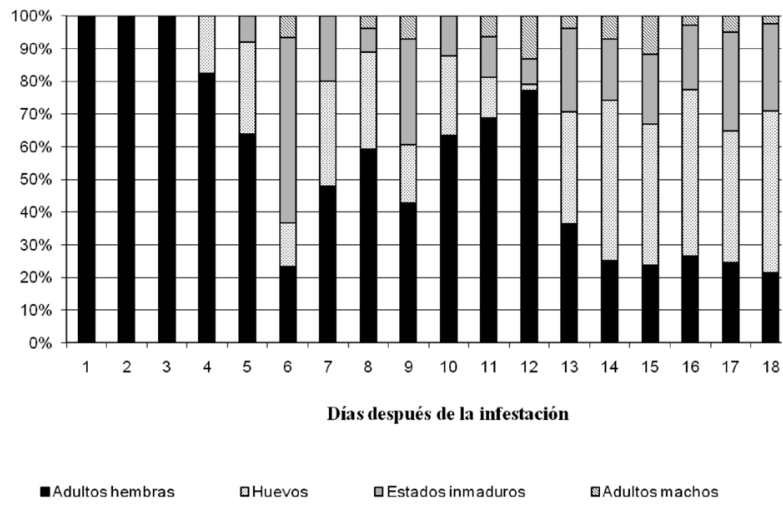

Figura 9. Estructura poblacional de $S$. spinki en $O$. latifolia bajo condiciones de invernadero en la Hacienda La Ligia, Puntarenas, Costa Rica. 2007.

La relación nominal entre machos y hembras de S. spinki encontrados, se muestra en el Cuadro 3. En el día 1 de evaluación se encontró que hubo un promedio 1,25 hembras/ planta, que colonizaron la especie $O$. sativa. Este valor fue superior a la colonización obtenida en $O$. latifolia. En el día 6 de muestreo en la especie $O$. latifolia se observó la aparición de machos que alcanzaron una densidad de 0,25 individuos/ planta, y una relación nominal de 1:3,52 (machos : hembras). La aparición de machos $S$. spinki en la especie O. sativa, se dio durante los días 12 al 18, con un máximo valor alcanzado de 2,2 hembras por cada macho observado. En la arvense O. latifolia, se evidenció que la cantidad de hembras con respecto a los individuos machos alcanzó valores de 9,0 hembras por cada macho observado, muy superior a lo hallado para $O$. sativa.

Cuadro 3. Proporción hembras: machos de $S$. spinki en dos fuentes alimenticias. Puntarenas. Costa Rica. 2007.

\begin{tabular}{ccc}
\hline Días de evaluación & \multicolumn{2}{c}{ Relación hembras : machos } \\
\cline { 2 - 3 } & 0. sativa & 0. latifolia \\
\hline 1 & $1,3: 0$ & $1,1: 0$ \\
6 & $3,0: 0$ & $3,5: 1$ \\
12 & $2,2: 1$ & $5,8: 1$ \\
18 & $2,0: 1$ & $9,0: 1$ \\
\hline
\end{tabular}




\section{SOBREVIVENCIA IN VITRO}

Ciclo de vida de S. spinki

La duración media del ciclo de vida de S. spinki desde huevo hasta adulto fue de siete días a temperatura de $25^{\circ} \mathrm{C}$. Se determinó que la duración mínima y máxima en el ciclo es de cuatro y diez días, respectivamente (Cuadro 4). Estos resultados representaron un paso inicial en los estudios de determinación del potencial reproductivo de esta importante plaga, pues constituyen los primeros que se obtuvieron para esta especie bajo las condiciones ambientales de Costa Rica.

Cuadro 4. Duración promedio (mínimos y máximos) en días del ciclo de vida de $S$. spinki, en la Hacienda La Ligia, Puntarenas, Costa Rica. 2007.

\begin{tabular}{cccc}
\hline Instar & Media & Mínimo & Máximo \\
\hline Huevo & 2,5 & 1,5 & 3,5 \\
Larva móvil & 2,5 & 1,5 & 3,5 \\
Larva inactiva & 2,0 & 1,0 & 3,0 \\
\hline TOTAL & $\mathbf{7 , 0}$ & $\mathbf{4 , 0}$ & $\mathbf{1 0 , 0}$ \\
\hline
\end{tabular}

Se observó para las tres fuentes alimenticias evaluadas, que la eclosión resultó notablemente afectada con pérdidas medias del $44 \%$ de los huevos. Aunque son similares las condiciones de hábitat y alimentación ofrecidas por $O$. sativa y $O$. latifolia, se pudo determinar que la especie arvense disminuyó en un $12 \%$ el porcentaje de individuos que pudieron alcanzar el estado adulto (Cuadro 5).
Cuadro 5. Mortalidad (\%) de S. spinki durante estadios del ciclo de vida evaluado en la Hacienda La Ligia, Puntarenas, Costa Rica. 2007.

\begin{tabular}{cccc}
\hline & \multicolumn{3}{c}{ Fuentes alimenticias } \\
\hline Instar & O. sativa & O. latifolia & E. colona \\
\hline Huevo & 36,0 & 38,0 & 58,0 \\
Larva móvil & 18,0 & 26,0 & 42,0 \\
Larva inactiva & 8,0 & 10,0 & 0,0 \\
\hline TOTAL & $\mathbf{6 2 , 0}$ & $\mathbf{7 4 , 0}$ & $\mathbf{1 0 0 , 0}$ \\
\hline
\end{tabular}

La fuente alimenticia $O$. sativa evidenció menor mortalidad en los diferentes estadios de desarrollo de $S$. spinki. La especie E. colona afectó totalmente la eclosión de huevos y el instar de larva móvil con mortalidad del $58 \%$ y $42 \%$ de los individuos, respectivamente. Es decir, la arvense no ofrece las condiciones mínimas de alimentación que pudieron permitir el desarrollo del ciclo de vida de S. spinki.

\section{DISCUSIÓNY CONCLUSIONES}

\section{SOBREVIVENCIA BAJO CONDICIONES DE INVERNADERO}

\section{Sobrevivencia}

El ensayo se realizó con plantas de $O$. sativa, $O$. latifolia y E. colona, en etapa de floración, con el propósito que $S$. spinki se desarrollara de mejor manera en la etapa siguiente de llenado de granos. Lo anterior se determinó con base en investigaciones de Chen et al. (1979), que concluyeron que el pico de población del ácaro se alcanzó cuando la plantación de arroz se encontraba en la fase de grano lechoso, en la que ocurre el transporte de los nutrimentos sintetizados. La metodología planteada tuvo como desventaja que no se realizaron estudios preliminares que determinen el momento fenológico que propicia el mejor desarrollo de las poblaciones de $S$. spinki en las arvenses evaluadas.

La colonización del ácaro en las tres plantas que se evaluaron, demostró la mayor 
preferencia de éste por $O$. sativa y $O$. latifolia, e insuficiente en E. colona. El momento de colonización determinó las características de los resultados encontrados posteriormente, pues se observó que la oviposición dependió de este factor. Cabe resaltar que a pesar de utilizarse dos metodologías al momento de infestación, solo se obtuvo colonización por parte de las hembras, mientras que para los machos se verificó la muerte en el sitio donde eran colocados, en la medida que se secaba el tejido vegetal portante del que se obtuvo la población para la infestación artificial.

Informes realizados por Almaguel (1996), indicaron que para S. spinki y otros ácaros plaga en Cuba, se presentó una disminución drástica debida a la mortalidad directa que provocó la lluvia, por efecto del lavado de adultos y estados inmaduros. El presente experimento bajo invernadero disminuyó la influencia directa de las precipitaciones sobre la sobrevivencia y crecimiento poblacional de $S$. spinki. Al realizar el riego con regadera manual, permitió disminuir el golpe directo sobre las vainas de las hojas superiores de las especies, que fue donde se hospedó la mayor población de ácaros.

En $O$. sativa se presentó un mínimo de 1,13 ácaros/ planta en los tres primeros días, y se alcanzó un máximo de 163,0 ácaros/ planta para el día 18 después de infestación. Es de mencionar, que durante el desarrollo del experimento se disminuyó la colonización favorecida por elementos como el aire, el agua, insectos y maquinaria. Se supone que los resultados obtenidos de crecimiento poblacional, se debieron solamente a la reproducción alta del ácaro. Con respecto a lo anterior, Ramos y Rodríguez (2001) han considerado que la tasa de establecimiento estuvo en función de la reproducción y de la dispersión, y han reportado en campo abierto promedios de 480,0 ácaros/ planta en la fase de inflorescencias. El crecimiento poblacional de $S$. spinki pudo relacionarse con las condiciones óptimas de hábitat y alimento ofrecidas por cada una las especies hospedantes, sin embargo, durante el presente estudio, no se pudo determinar con certeza la mencionada relación.
Durante la etapa de establecimiento de S. spinki sobre las vainas de las hojas de $O$. sativa, se observaron huevos desde el momento de la migración, mientras en $O$. latifolia fue necesario que transcurrieran aproximadamente 72 horas para que el ácaro encontrara condiciones adecuadas para la oviposición. Este aspecto disminuyó la densidad poblacional observada posteriormente.

Con respecto a E. colona, se observó migración del ácaro y presencia ocasional de bajo número de huevos. Se evidenció que de los huevos emergieron larvas móviles que no encuentran condiciones óptimas para su alimentación, probablemente por la dureza de los tejidos de la arvense.

Hasta el momento no existen trabajos que expliquen el comportamiento preciso del ácaro en la planta arvense hospedante $E$. colona. El presente trabajo permitió concluir que el ácaro solo utilizó a E. colona como planta hospedante para individuos hembras adultas, con el siguiente proceso: colonización, oviposición, eclosión de huevos y nacimiento de larva móvil. Lo anterior se concluyó por las condiciones físicas identificadas en algunas larvas encontradas, que se observaronn delgadas y fláccidas; y por el excesivo movimiento que indicaba la necesidad de escapar.

\section{Crecimiento poblacional}

En la especie arvense $O$. latifolia el ácaro presentó durante los tres períodos de análisis, tasas de crecimiento superiores a las encontradas para $O$. sativa. Lo anterior se relacionó posiblemente con el tiempo en el cual las plantas que se utilizaron en el experimento, cambiaron de etapa fenológica. Al iniciar el experimento, las plantas de las tres especies evaluadas, en cada parcela y cada uno de los bloques establecidos, se encontraban en la fase de embuchamiento, pero al cabo de una semana se observó que el $60 \%$ de las plantas de O. latifolia presentaron emersión de la panícula. Lo anterior permitió suponer que durante el ensayo la especie arvense brindó condiciones alimenticias 
óptimas (transporte de nutrimentos), que se reflejaron en el crecimiento poblacional del ácaro. El análisis anterior se realizó con base en los resultados obtenidos por Chen et al. (1979), Lo y Ho (1979), quienes indicaron que el pico de la población del ácaro se obtuvo cuando las plantaciones alcanzan la fase de grano lechoso.

Las plantas arvenses utilizadas como hábitat para $S$. spinki durante el experimento fueron tomadas de campos arroceros un mes después de la cosecha, en los que han emergido plantas invasoras y se encontraban en desarrollo; y las de arroz comercial se extrajeron de un campo establecido hacía 45 días después de siembra. Durante la etapa experimental no se incluyó el plan de fertilización, por lo que se supone que este factor disminuyó la calidad nutricional de todas las plantas evaluadas y por lo tanto, provocó disminución en el potencial biótico que podría desarrollar el ácaro bajo condiciones ideales de alimentación.

En la actualidad no se tienen investigaciones en Costa Rica, que permitan determinar el grado de susceptibilidad de las variedades de $O$. sativa frente al ataque de S. spinki. En Cuba, la evaluación realizada por Botta et al. (2003) del comportamiento de S. spinki en diferentes variedades de arroz, concluyó que especies altamente susceptibles como Perla de Cuba, albergan hasta 78,3 ácaros/ planta. Las especies de mejor comportamiento como IACuba-28, presentaron medias de 1,0 ácaro/ planta, y las variedades de comportamiento susceptible medio como Reforma y IACuba-27 presentaron densidades de entre 10,1 y 18,3 ácaros/ planta. De acuerdo con lo anterior, y al observar que se encontraron en promedio 163,0 ácaros/ planta a los 18 días después de infestación, pudo concluirse que $O$. sativa var. Palmar 18, presentó alta susceptibilidad al ser atacada por S. spinki.

La insuficiente información existente con respecto a la relación ácaro-variedades cultivadas en Costa Rica, hizo necesario el diseño de experimentos que permitieran comparar la susceptibilidad de las distintas variedades cultivadas bajo las condiciones agroecológicas del país. La metodología experimental empleada en el presente trabajo pudo ser utilizada en los diseños de los estudios propuestos.

Teniendo en cuenta el análisis de sobrevivencia y crecimiento poblacional de $S$. spinki, se concluyó que esta solo presenta como planta hospedante alterna en los campos de arroz en Costa Rica, la especie arvense O. latifolia; y E. colona es una planta hospedante ocasional que permitió el refugio transitorio por cortos períodos de tiempo.

\section{Estructura poblacional}

Bajo condiciones de laboratorio, Lo y Ho (1979) obtuvieron una relación sexual hembra-macho de $8: 1$ con temperaturas de 29 +/- 3ํㅡ. Al comparar los resultados obtenidos por estos autores y los obtenidos en la presente investigación, se pudo concluir que hubo diferencias considerables en cuanto a la relación nominal hembras : machos con respecto a la variedad comercial Palmar 18 que se utilizó en el experimento. Sin embargo, pudo observarse que la arvense $O$. latifolia en el día 18 de evaluación permitió determinar una relación similar a la informada en literatura. Este fenómeno se pudo explicar por el cambio ocurrido en la etapa fenológica de $O$. latifolia, en la que pudo observarse la formación de semillas; mientras $O$. sativa permaneció en etapa de embuchamiento durante todo el experimento. La etapa alcanzada por la planta arvense, posiblemente provocó una población final bien conformada de ácaros y un mayor número de machos.

\section{SOBREVIVENCIA IN VITRO}

\section{Ciclo de vida de S. spinki}

Tseng (1985) informó para Taiwán que la duración del desarrollo de esta especie oscila entre 16 y 17 días a $25^{\circ} \mathrm{C}$. Al comparar los resultados de este autor con los encontrados para las condiciones ambientales de la zona 
arrocera Pacífico Central, se apreció que el ciclo de vida del ácaro presenta disminución de un $43,8 \%$, lo que lleva a concluir que $S$. spinki encontró en esta zona las condiciones óptimas de clima y que la variedad de arroz comercial Palmar 18 presentó alta susceptibilidad de ser atacada por la plaga.

Los resultados obtenidos en la presente investigación se aproximan a los informados por Ramos y Rodríguez (2001) para las condiciones ambientales de Cuba, quienes informaron que la duración en el ciclo de vida varió entre 5 y 9 días desde huevo hasta adulto a $24,4^{\circ} \mathrm{C}+/-1,1^{\circ}$.

La metodología que se empleó para la evaluación in vitro evidenció, una alta pérdida de huevos de S. spinki, por lo que se requirieron modificaciones para nuevos experimentos, pues fue evidente la desecación de los huevos al estar expuestos a las secciones de vainas de hojas. Durante los estadios de larva móvil, larva inactiva y adultos, fue necesario cambiar las secciones vegetales desecadas cada 12 horas, lo cual modificó el comportamiento natural del ácaro e influyó sobre la mortalidad evidenciada en el experimento.

Con respecto a las características nutricionales ofrecidas por cada especie vegetal evaluada, se concluyó que $E$. colona solo sirve de hospedante ocasional y no garantiza el éxito de las larvas móviles, por lo que las prácticas de manejo del ácaro en esta arvense deben diseñarse para combatir la presencia de hembras adultas. Por el contrario, la arvense O. latifolia ofreció condiciones nutricionales para la reproducción y desarrollo del ciclo de vida de S. spinki.

Teniendo en cuenta los resultados obtenidos en el presente estudio, se pudo concluir que en aquellos campos arroceros con alta presencia de $O$. latifolia, el ácaro encuentra condiciones ideales para su reproducción durante todos los meses del año. Es por ello, que deben continuarse trabajos de investigación enfocados al manejo eficiente de esta planta arvense y a la planificación estratégica de las prácticas agrícolas, debido a la estrecha relación $O$. latifolia - $S$ spinki.

\section{AGRADECIMIENTOS}

Los autores agradecen a las instituciones costarricenses: Instituto Nacional de Innovación y Transferencia de Tecnología Agropecuaria (INTA), Corporación Arrocera Nacional (CONARROZ), y al personal administrativo y de campo de la Hacienda La Ligia, por su enorme contribución a las labores de establecimiento y muestreo durante el experimento.

\section{LITERATURA CITADA}

Almaguel, L.; Santos, A.; Torre, P.E. de la; Botta, E.; Hernández, J.; Cáceres, I.; Ginarte, A. 2003. Dinámica de población e indicadores ecológicos del ácaro Steneotarsonemus spinki Smiley 1968 (Acari: Tarsonemidae) en arroz de riego en Cuba. Fitosanidad 7(1): 23-30.

Almaguel R., L. 1996. Ácaros de importancia económica en Cuba. Boletín técnico no. 2. La Habana, CID-INISAV.

Anónimo. 2005. Dinámica poblacional. Modelos de crecimiento. Disponible en: http://www.virtual.unal.edu. co/cursos/agronomia/2005840/ lecciones/cap04/Lec4_2.htm. Consultado el 22 de noviembre de 2007. Hora: 8:00 a.m.

Barquero S., M. 2004. Limón y Guanacaste: Severo ataque de ácaro del arroz. La Nación, San José, CR, agosto. 3.

Botta, E.; Almaguel, L.; González, T.; Arteaga, I.; Hernández, J. 2003. Evaluación del comportamiento de Steneotarsonemus spinki en diferentes variedades de arroz durante los años 2000-2001. Fitosanidad. 7(2): 25-29. 
Correa-Victoria, F. 2006. Complejo acarohongo-bacteria del arroz. Centro Internacional de Agricultura Tropical (CIAT). Disponible en: http://www. ciat.cgiar.org/riceweb/esp/pdf/ complejo_acaro_costa_rica.pdf. Consultado el 23 de noviembre de 2007. Hora: 10:00 a.m.

Chen, C.N.; Cheng, C.C., Hsiao, K.C. 1979. Bionomics of Steneotarsonemus spinki attacking Rice Plants on Taiwan. Recent Advances in Acarology. 1: 111-117.

FAO. 2006. Compendio de indicadores relativos a la agricultura y la alimentación. Disponible en:http://www.fao.org/ es/ess/es/compendium_2006/ technotes.asp. Consultado el 10 de noviembre de 2007. Hora: 5:00 pm.

Holdridge, LR. 1993. Mapa ecológico de Costa Rica según el sistema de clasificación de zonas de vida del mundo. Centro Científico Tropical (CCT)/ Ministerio de Agricultura y Ganadería (MAG).

Kang-Chen, L.; Chyi-Chen, H. 1979. Screening of chemicals for the control of rice Tarsonemid mite Steneotarsonemus spinki. J. Agric. Res. China. 30(3): 303-307.

Lo, Ch.K.; Ho, Ch.Ch. 1979. Ecological observations on Rice Tarsonemid Mite, Steneotarsonemus spinki (Acarina: Tarsonemidae). J. Agric. Res. China. 28(3): 181-192.

Ramos, M.; Rodríguez, H. 2001. Identificación y bioecologia de una nueva plaga en el arroz en Cuba. Revista MIPCATIE. 61: 43-53.

Ramos, M.; Rodríguez, H. 1997. Steneotarsonemus spinki Smiley (Acari: Tarsonemidae): nuevo informe para Cuba. Rev. Protección Veg. 13(1): 25-28.
Sandoval, R. 1998. Consideraciones sobre la enfermedad de la pudrición de la vaina del arroz por Sarocladium oryzae (Sawada) Gams \& Hawks. I Encuentro Internacional de arroz. Ciudad de la Habana, Cuba.

Santos, A.; Almaguel, L.; Torre, $\mathrm{P}$ de la; Cortiñas, J.; Cáceres, I. 1998. Duración del ciclo de vida en condiciones controladas del ácaro Steneotarsonemus spinki (Acari: Tarsonemidae) en arroz (Oryza sativa L.) en Cuba. I Encuentro Internacional de Arroz. Palacio de Convenciones, La Habana. p. 187.

Smiley, R.L.; Flechtmann, CH.W.; Ochoa, R. 1993. A new species of Steneotarsonemus (Acari: Tarsonemidae) and an illustrated key to grass-infesting species in the Western Hemisphere. Internat. J. Acarol. 19(1): 87-1993.

Tseng, Y.H. 1985. Mites associated with weeds, paddy rice and upland rice fields in Taiwan. Acarology. VI (2): 770-780. 